\title{
Texture Representation of SAR Sea Ice Imagery Using Multi-Displacement Co-Occurrence Matrices
}

\author{
Leen-Kiat Soh and Costas Tsatsoulis \\ Department of Electrical Engineering and Computer Science \\ University of Kansas, Lawrence, Kansas \\ address: Center for Excellence in Computer-Aided Systems Engineering \\ 2291 Irving Fill Road, Lawrence, KS 66045 \\ tel: (913)864-7767 fax: (913)864-7787 e-mail: lksoh@ cecase.ukans.edu
}

\begin{abstract}
In this paper, we desribe multi-displacement cooccurrence matrices for representing sea ice textures of SAR imagery. Our design of co-occcurrence matrices captures local relationships among neighboring pixels and global links among distant pixels, an advantage over other existing versions of co-occurrence matrices. As a result, it can adequately represent micro textures, such as grainy details, and macro textures, such as patchy blocks. We have conducted experiments to compare our multi-displacement co-occurrence matrices with other existing versions using Bayesian linear discrimination. We have found that our design is the most texturally representative in terms of classification accuracies in both training and test datasets. In addition, we have applied this design to sea ice texture analysis which includes detection and localization, and subsequent image-texture mapping.
\end{abstract}

\section{INTRODUCTION}

Statistical texture analysis has been important in SAR sea ice imagery research since, with it, sea ice regions can be better represented and thus classified, compared to analysis based on only intrinsic gray levels. For example, [1] classified one SAR image (over the Beaufort Sea) to new/first-year ice and multiyear ice with an overall accuracy of more than $65 \%$ using derived textural descriptors on $\mathrm{X}$ band (HV polarization). Standard statistics and higher order texture statistics generated from co-occurrence matrices were used to classify SAR sea ice data with an overall accuracy of $89.5 \%$ [2]. Statistical textures have also been used in classifying other sea ice imagery such as Landsat Thematic Mapper (TM) Antarctic scenes [3]. In this paper, we concentrate on the statistical textural contexts of SAR sea ice regions for identifying structural composition of ice-water patterns, instead of surfacial textures that have been used for determining ice types.

We have chosen the gray level co-occurrence method as our texture analysis basis for three reasons. First, perceptual psychology studies [4] have shown this method to match a level of human perception. Second, studies $[5,6]$ have shown this method to outperform the others in texture discrimination. Third, co-occurrence matrices have been used successfully in many applications [7-10] and also in SAR imagery classification $[11,12]$.

In this paper, we present a study of different designs of cooccurrence matrices on SAR sea ice imagery. We investigate

This work is supported in part by NASA Grant NAGW-3043 the effects of the quantization and displacement, factors on implementing co-occurrence matrices for SAR sea ice imagery. We also present a classification experiment using Bayesian linear discrimination that compares different implementations of co-occurrence matrices. Finally, we apply the best implementation on SAR sea ice imagery to generate textural maps.

\section{BACKGROUND ON CO-OCCURRENCE MATRICES}

The original definition of gray-level co-occurence matrices is shown in [7]. Briefly, the texture-context information is specified by the matrix of relative frequencies $P_{i j}$ with two neighboring pixels separated by distance $d$ occur on the image, one with gray level $i$ and the other with gray level $j$. Such matrices of gray level co-occurrence frequencies are a function of the angular relationship and distance between the neighboring pixels. To implement co-occurrence matrices, one needs to identify the number of quantization levels, the orientation and displacement factors, given which one can determine the most representative co-occurrence matrix for certain application. An algorithm for selecting the matrix with the highest $\chi^{2}$ value was proposed such that one can determine the displacement and orientation parameters of the optimal matrix for classification [13].

\section{EXPERIMENTS}

Our experiments were designed to assess the best quantization and displacement values for representing SAR sea ice textures. Before conducting the experiments, we identified seven sea ice texture types: 1) Web-like, where the image consists of mostly multiyear ice with high ridge content, 2) High-deformation, where crushing of ice floes creates extreme deformations, usually found at marginal ice zones (MIZs), 3) Fractal-like, where the image consists mostly of new ice and melt ponds, especially at the end of summer melt season, 4) Pebble-like, where tiny round floes are embedded in younger ice formations, at the start of summer melt season, 5) Smooth, where floes are minimally deformed, with low ridge content, 6) High-contrast, where large dark multiyear ice floes and large refrozen young and thin pancake ice coexist, resulting from mobile floes that create water lodgings and yet are stationary enough for pancake or young ice to form, and 7) Packed, where the image consists of packed multi year ice occasionally broken up by leads. Beside their geophysical implications, these texture types were identified also because of their frequent 
occurrences during a study of a database of about 2000 SAR sea ice images. We conducted three experiments as follows:

\section{Experiment 1: Number of Quantization Levels}

This experiment used uniform quantization. We extracted sample sites, each $64 \times 64$, from 18 images with different textural regions. We devised a test using four textural features (energy, contrast, entropy, and homogeneity) and six different quantization schemes: $8,16,32,64,128$, and 256 levels. The displacement was 1 and orientations were $0^{\circ}$, $45^{\circ}, 90^{\circ}$, and $135^{\circ}$. Taking the average of the orientations yields for each sample site a two-dimensional vector. The degrees of similarity or disimilarity among samples, as derived from the vectors, were more stable across higher number of quantization levels, indicating that we should not use small number of quantization levels.

\section{Experiment 2: Displacement Parameter}

The displacement, $d$, is important in the computation of cooccurrence matrices. Applying a large displacement value to a fine texture would yield a co-occurrence matrix that does not capture the textural information, and vice versa. For our experiment, we used $d=1, \ldots, 32$. As a result, we generated a curve for each textural feature, for each quantization scheme. We concluded that across quantization schemes, each textural curve preserves nicely, indicating that it is sufficient to use one quantization scheme with a range of displacement values since the dynamics of the curves are similar.

Experiment 3: Implementations and A Comparative Study of Co-Occurrence Matrices

Experiments 1 and 2 provided us some ideas on designing a general co-occurrence matrix to represent SAR sea ice textures. Combining the conclusions of the experiments, we conjectured that a co-occurence matrix with a 64-level quantization and a range of displacement values should sufficiently and efficiently represent textures in sea ice. For SAR sea ice imagery, there are no systematic patterns based on orientation: ice floes position themselves in all possible orientations. We thus used $0^{\circ}, 45^{\circ}, 90^{\circ}$, and $135^{\circ}$. In this experiment, we had three implementations of co-occurrence matrices. The first implementation was called the mean displacement and mean orientation (MDMO) matrix. Textural measures are averaged over orientation and displacement values. This design assumes that every matrix of specific displacement and orientation values is partially representative for each region; and that the third order measurement, the average of the textural curve values, is constructive. The second implementation was called the $\chi^{2}$. optimal displacement and mean orientation (ODMO) matrix. $\chi^{2}$ values of all 4 matrices of different orientations are calculated and averaged for each displacement value, and the matrix accumulating the most $\chi^{2}$ value is the optimal matrix. This design assumes that only the matrix whose $\chi^{2}$ value is the highest with specific displacement is truly and sufficiently representative for the sample. The third one is called the $\chi^{2}$ optimal displacement and $\chi^{2}$-optimal orientation (ODOO) matrix. This design assumes that the matrix whose $\chi^{2}$ value is the highest with specific displacement and orientation is truly and sufficiently representative for the sample. To study these three implementations comparatively, we used a Bayes classifier. A Bayes classifier estimates covariance matrices from the different classes of training dataset and generates classifying rules. In testing, each rule will be used to compare an unknown instance to a certain class. The rule that yields the largest probability of membership gives the class of the unknown instance.

First, we used 240 sample sites, and seven texture groups. After training, we obtained seven classifying rules. We then re-applied all the samples as unknown instances to the rules, and the resubstitution or training set classification accuracies of the MDMO, ODMO, and ODOO implementations were $90.79 \%, 62.28 \%$, and $51.75 \%$, respectively. This exercise tells us that MDMO has the best capability in creating the necessary inter-class decision boundaries among the seven texture groups Second, to test the generality of our MDMO Bayes classifier, we divided the data set into two, trained the classifier on one and applied the trained classifier to the other. The training and test set classification accuracies were $99.19 \%$ and $94.17 \%$, respectively. We have concluded from these experiments: 1) The MDMO implementation is the most representative of SAR sea ice textures, 2) The range of displacement values as a whole is more representative than a displacement value alone. This indicates that MDMO, using a third order measurement (i.e., the average of the curve), is able to capture local and global details of a texture, and 3) Matrices with the highest $\chi^{2}$ value did not provide useful information. We named the MDMO implementation MultiDisplacement Co-Occurrence Matrix.

\section{RESULTS}

We have applied Multi-Displacement Co-Occurrence Matrix to generate texture maps of SAR sea ice imagery, thereby detecting and locating regions with high/low ridge content, high/low deformation, high/low melt, etc. For example, Fig. 1 shows an original image and Fig. 2 shows a texture classification of the image: $53.62 \%$ Web-like, 31.26\% Fractal-like, $10.79 \%$ Pebble-like, $7.79 \%$ Smooth, and $3.56 \%$ High-contrast. The texture classification and mapping results have been encouraging, and our planned future work includes gathering more diversified test sets and improving the classification power of our co-occurrence matrix.

\section{CONCLUSIONS}

We bave implemented a texture representation technique for SAR sea ice imagery and shown that it has more classification power than co-occurrence matrix that uses only a single displacement value. We have also shown that a straight 64-level quantization scheme is able to discriminate different textures adequately. In addition, we have also applied this design to SAR sea ice imagery which includes 
localization of surface deformation, high ridge content, and melt pond regions, which is useful in sea ice geophysical analysis.

\section{REFERENCES}

[1] Q. A. Holmes, D. R. Nuesch, and R. A. Shuchman, "Textural Analysis and Real-Time Classification of SeaIce Types Using Digital SAR Data," IEEE Transactions on Geoscience and Remote Sensing, vol. 22, no.2, pp. 113-120, 1984.

[2] J. A. Nystuen and F. W. Garcia, Jr., "Sea Ice Classification Using SAR Backscatter Statistics," IEEE Transactions on Geoscience and Remote Sensing, vol. 30, no. 3, pp. 502-509, 1992.

[3] J. Chou, R. C. Weger, J. M. Ligtenberg, K.-S. Kuo, R. M. Welch, and P. Breeden, "Segmentation of Polar Scenes Using Multi-Spectral Texture Measures and Morphological Filtering," International Journal of Remote Sensing, vol. 15, no. 5, pp. 1019-1036, 1994.

[4] B. Julesz, E. N. Gilbert, L. A. Shepp, and H. L. Frisch, "Inability of Humans to Discriminate Between Visual Textures that Agree in Second-Order StatisticsRevisited," Perception, vol. 2, pp. 391-405, 1973.

[5] J. S. Weszka, C. R. Dyer, and A. Rosenfeld, "A Comparative Study of Texture Measures for Terrain Classification," IEEE Transactions on Systems, Man, and Cybemetics, vol. 6, pp. 269-285, 1976.

[6] R. W. Conners and C. A. Harlow, "A Theoretical Comparison of Texture Algorithms," IEEE Transactions on Pattern Analysis and Machine Intelligence, vol. 2, no. 3, pp. 204-222, 1980.

[7] R. M. Haralick, K. Shanmugan, and I. H. Dinstein, "Textural Features for Image Classification," IEEE Transactions on Systems, Man, and Cybernetics, vol. 3, pp. $610-621,1973$.

[8] R. P. Kruger, W. B. Thompson, and A. F. Turner, "Computer Diagnosis of Pheumoconiosis," IEEE Transactions on Systems, Man, and Cybernetics, vol. 4, no. 1, pp. 40-49, 1974.

[9] Y. P. Chien, and K. -S. Fu, "Recognition of X-Ray Picutre Patterns," IEEE Transactions on Systems, Man, and Cybemetics, vol. 4, no. 2, pp. 145-156, 1974.

[10] S. P. S. Kushwaha, S. Kuntx, and G. Oesten, "Applications of Image Texture in Forest Classification," International Journal of Remote Sensing, vol. 15, no. 11, pp. 2273-2284, 1994.

[11] K. Shanmugan, V. Narayanan, V. S. Frost, J. A. Stiles, and J. C. Holtzman, "Textural Features for Radar Image Analysis," IEEE Transactions on Geoscience and Remote Sensing, vol. 19, no. 3, pp. 153-156, 1981.

[12] D. G. Barber and E. F. LeDrew, "SAR Sea Ice Discrimination Using Texture Statistics: A Multivariate Approach," Photogrammetric Engineering and Remote Sensing, vol. 57, no. 4, pp.385-395, 1991.

[13] S. W. Zucker and D. Terzopoulos, "Finding Structure in Co-Occurrence Matrices for Texture Analysis,"
Computer Graphics and Image Processing, vol. 12, pp. 286-308, 1980.

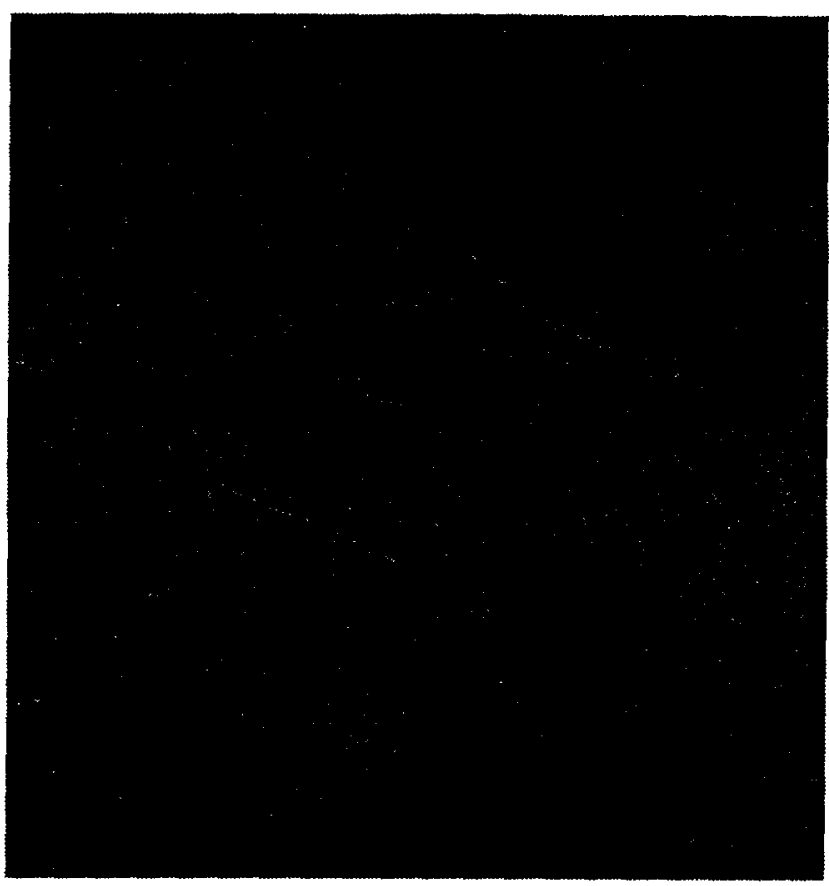

Fig.1 The orignal SAR image, Mar 27,1992 , at $73.46^{\circ} \mathrm{N}$, $156.19^{\circ} \mathrm{E}$. Copyright ESA.

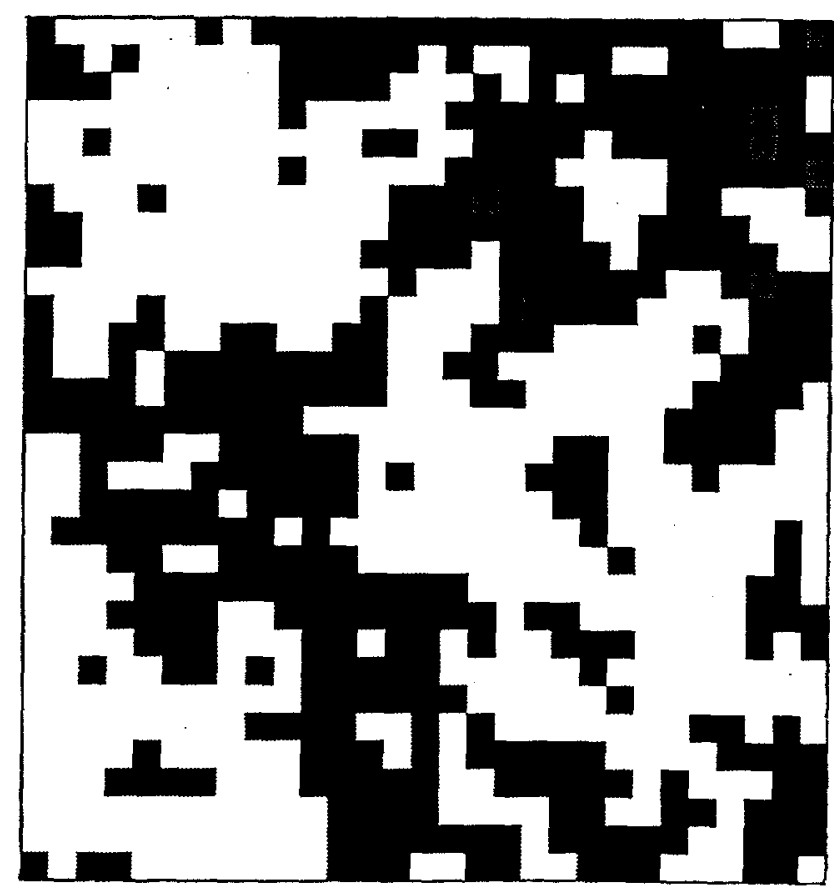

Fig.2 The texture map of Fig.1. More than half (bright regions) of the image has been classified as Web-like. 